\title{
The in vitro evaluation of isolated procyanidins as modulators of cytokine-induced eotaxin production in human alveolar epithelial cells
}

\author{
Sara L. Coleman ${ }^{\mathrm{a}, \mathrm{b}, *}$, Roger D. Hurst ${ }^{\mathrm{a}}$, Gregory M. Sawyer ${ }^{\mathrm{a}}$ and Marlena C. Kruger ${ }^{\mathrm{b}}$ \\ ${ }^{a}$ Food and Wellness Group, The New Zealand Institute for Plant \& Food Research Ltd, Palmerston North, \\ New Zealand \\ ${ }^{\mathrm{b}}$ Massey University Institute of Food Science and Technology, School of Food and Nutrition, Palmerston North, \\ New Zealand
}

Received 8 December 2015; accepted 9 February 2016

\begin{abstract}
.
BACKGROUND: Populations that consume procyanidin-rich diets are less susceptible to inflammatory disease. Allergic asthma is an inflammatory lung disease perpetuated by a hyperreactive airway epithelium and eosinophil infiltration into the lung. Eotaxin-1 (CCL11) mediates eosinophil migration into tissues and its modulation could represent a means to assist the management of airway inflammation.

OBJECTIVE: Here we evaluated procyanidins as a means of modulating CCL11 production in vitro.

METHODS: We used human lung epithelial cells (A549) and optimized the conditions to induce CCL11 production in vitro. Cells were exposed to procyanidins for $6 \mathrm{~h}$ prior to an inflammatory insult of $5 \mathrm{ng} / \mathrm{mL} \mathrm{IL}-4$ with $5 \mathrm{ng} / \mathrm{mL}$ TNF $\alpha$ for $48 \mathrm{~h}$. An enzyme-linked immunosorbent assay was used to measure CCL11 production.

RESULTS: Cells exposed to $5 \mu \mathrm{M}$ procyanidin A2 prior to the inflammatory challenge showed significantly inhibited (36\%) CCL11 production. Under the same conditions, procyanidins B1 and B2 elicited no effect. Furthermore, combinations of procyanidins A2 and B2 ( $5 \mu \mathrm{M}$ total) demonstrated no evidence of a synergistic interaction.

CONCLUSIONS: These data demonstrate that the regulation of CCL11 by lung epithelial cells is not ubiquitous among the three investigated procyanidins. We demonstrate a differential inhibition of CCL11 by A-type and B-type procyanidins. This evidence supports further studies into procyanidins, specifically A-type, for managing inappropriate airway inflammation.
\end{abstract}

Keywords: A549, eotaxin-1 (CCL11), IL-4, lung inflammation, procyanidins

\footnotetext{
*Corresponding author: Sara L. Coleman, The New Zealand Institute for Plant \& Food Research Limited, Private Bag 11600, Palmerston North 4442, New Zealand. Tel.: +64 6355 6178; E-mail: sara.coleman@plantandfood.co.nz. 


\section{Introduction}

Epidemiological evidence has revealed populations that consume procyanidin-rich diets are less susceptible to inflammatory disease, such as asthma [1,2]. Asthma is an inflammatory lung disease that involves inappropriate airway inflammation, bronchiole constriction, and lung tissue remodelling that collectively restrict airflow [3]. In allergic asthma these pathophysiological features are perpetuated by Th2 cell driven mechanisms and the infiltration of immune cells into lung tissue [4]. During homeostasis, eosinophil migration into the lung tissue would be tightly controlled; however, the hyperreactive airway epithelium of an asthmatic produces the chemokine eotaxin (CCL11 and its associated isoforms CCL24, and CCL26) in excess, which along with other cytokines instigates the allergic cascade [5].

Procyanidins are polyphenolic secondary plant metabolites that are thought to be biologically active in the context of human health. They are the product of polymerization of monomeric flavon-3-ol units by a currently unknown mechanism within plants [6]. The monomeric units can be bonded together by either A-type or B-type linkages. The differences in structure that result due to linkage are suspected to play a role in the biological activity of procyanidins. In the western diet, fruit are the predominate source of dimeric procyanidins, compounds that consist of two flavon-3-ol units [7]. Cranberries and lingonberries are high in A-type bonded procyanidins such as structurally-defined, procyanidin A2, whereas, apples are a high source of B-type bonded procyanidins such as structurally-defined procyanidin B1 and procyanidin B2 [8]. Polyphenolic metabolites are thought to accumulate in plants as a consequence of stress or pathogenic infiltration [9]. Modern agriculture limits these stresses; Joven et al. suggested that in a western diet it would be difficult to consume enough polyphenols from fruit and vegetables to influence health as a result of reduced polyphenolic profiles due to modern agricultural practices $[10,11]$. Furthermore, the mean consumption of fruit and vegetable servings per person in the US is half of the recommended portions at 1.5-2 cups and 2-3 cups, respectively, per day [12]. Thus, concentrated extracts or isolated compounds could be incorporated into foods to supplement the typical western diet for improved health and wellbeing.

In this study, we investigated commercially sourced, structurally-defined, pure dimeric procyanidin compounds for in vitro efficacy of modulating one key biomarker, CCL11, relevant in allergic asthma. Understanding the role of procyanidins in managing inflammation could allow for their use in fresh or processed foods as a natural means to prevent and manage inflammatory illness, limit the use of pharmaceutical interventions, and assist with improving human health.

\section{Materials and methods}

\subsection{Materials}

The human alveolar epithelial (A549) cell-line was purchased from the American Tissue Cell Collection (ATCC ${ }^{\circledR}$ CCL-185 ${ }^{\mathrm{TM}}$ (c/o Cryosite Lane Cove, NSW, Australia)). Cell culture media, phosphate buffered saline (PBS), penicillin-streptomycin-neomycin antibiotic mixture, 100x L-glutamine, and 2.5\% trypsin were purchased from Life Technologies (Auckland, NZ). Fetal bovine serum (FBS) was purchased from Moregate Biotech (Hamilton, NZ). Procyanidin A2 (HPLC $\geq 99 \%$, epicatechin-(4 $\beta-8,2 \beta-0-7)$-epicatechin), procyanidin B1

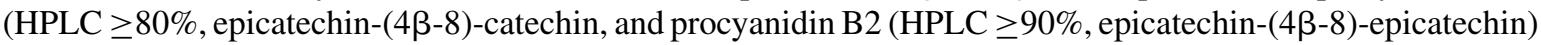
were purchased from Extrasynthese (Genay, France). Human recombinant IL-4, TNF $\alpha$, IFN $\gamma$, and CCL11 DuoSet ELISA kits were purchased from R\&D Systems (Pharmaco, Auckland, NZ). Low-endotoxin bovine serum albumin was purchased from MP Biomedicals (Auckland, NZ). WST-1 reagent was purchased from Roche (Auckland, NZ). Dimethyl sulfoxide (DMSO) and all other chemicals not specifically listed were purchased from Sigma-Aldrich (St. Louis, Missouri, USA). 


\subsection{Isolated procyanidins}

Procyanidins were dissolved in DMSO and stored at $-80^{\circ} \mathrm{C}$ until experimental use. Procyanidins were then dissolved in serum-free media to desired concentration and added immediately to cell cultures. In synergy experiments concentrations of each procyanidin were prepared separately and added together in the cell culture well. The final DMSO concentration was $0.58 \%$ and $1.15 \%(\mathrm{v} / \mathrm{v})$ DMSO respectively for cultures exposed to $5 \mu \mathrm{M}$ and $10 \mu \mathrm{M}$ procyanidins and were used in the negative controls.

\subsection{Cell culture conditions}

Cells were grown under standard tissue culture conditions of $37^{\circ} \mathrm{C}$ in a $95 \%$ humidified atmosphere at $5 \% \mathrm{CO}_{2}$ in Dulbecco's Modified Eagle Medium: Nutrient Mixture F-12 (DMEM/F-12) containing 2 mM L-glutamine, $50 \mu \mathrm{g} / \mathrm{mL}$ penicillin, $50 \mu \mathrm{g} / \mathrm{mL}$ streptomycin, $100 \mu \mathrm{g} / \mathrm{mL}$ neomycin, and $10 \%$ FBS. The cell cultures were grown to form a monolayer ( $80 \%$ confluence) and then growth arrested for $24 \mathrm{~h}$ in the absence of FBS before conducting experiments. Experiments used A549 cells of passage 89-107.

\subsection{Cytotoxicity}

Cell metabolism (viability/ cytotoxicity) after $6 \mathrm{~h}$ procyanidin exposure was measured using water soluble tetrazolium-1 (WST-1) reagent to ensure isolated procyanidins were not cytotoxic to cell cultures [13]. During the WST-1 assay, the rate at which tetrazolium salts are cleaved to formazan by cellular mitochondrial dehydrogenases correlates with the number of viable cells in the culture. Changes in the formation of formazan in response to procyanidins is extrapolated to cellular viability/cytotoxicity. A549 cells were plated at $5 \times 10^{4}$ per well in 96well plates with media changes to serum-free media $24 \mathrm{~h}$ before starting the experiment. Cells were incubated with control media, positive control $2.5 \mathrm{mM} \mathrm{H}_{2} \mathrm{O}_{2}$, or procyanidin $\mathrm{B} 1, \mathrm{~B} 2$, or $\mathrm{A} 2$ at concentrations ranging from $1 \mu \mathrm{M}$ to $20 \mu \mathrm{M}$ for $6 \mathrm{~h}$. After a wash with PBS, WST-1 assay reagent was added to each well and cellular viability was measured according to the manufacturer's directions.

\subsection{Inducing the production of CCL11}

The production of CCL11 can be induced in A549 airway epithelial cells in vitro to model the inappropriate inflammation that occurs during allergic asthma. Production of CCL11 is both concentration- and time-dependant. Other work by our group characterized the concentration- and time-dependant profile of IL-4 on the production of CCL26, another isoform of eotaxin, generated by A549 cells [14]. IL-4 and TNF $\alpha$ exposure alone were insufficient to substantially induce CCL11 (data not shown). Thus, we evaluated CCL11 production when exposed to $5 \mathrm{ng} / \mathrm{mL}$ IL-4 with a range of TNF $\alpha$ concentrations from $0.75 \mathrm{ng} / \mathrm{mL}$ to $40 \mathrm{ng} / \mathrm{mL}$ for $48 \mathrm{~h}$, as well as, determined the time course of CCL11 production after exposure to $5 \mathrm{ng} / \mathrm{mL} \mathrm{IL-4}$ with $5 \mathrm{ng} / \mathrm{mL} \mathrm{TNF} \alpha$ for times ranging from $1-72 \mathrm{~h}$. Experiments used A549 cells plated at $5 \times 10^{5}$ per well in 12-well plates with media changes as described above. Cells were then exposed to serum-free media containing the cytokines. Extracellular media was collected and frozen at $-80^{\circ} \mathrm{C}$ until analysed for CCL11 by ELISA.

\subsection{Enquiry into procyanidin efficacy}

In experiments examining effects of procyanidins on inflammation, A549 cells were plated at $5 \times 10^{5}$ per well in 12-well plates with media changes as described above. Cells were then incubated with media containing procyanidins at concentrations ranging from $1 \mu \mathrm{M}$ to $10 \mu \mathrm{M}$, carrier control DMSO, or positive control $5.8 \mu \mathrm{M}$ IFN $\gamma$ for $6 \mathrm{~h}$, washed with PBS, and then stimulated with $5 \mathrm{ng} / \mathrm{mL} \mathrm{IL-4} \mathrm{and} 5 \mathrm{ng} / \mathrm{mL} \mathrm{TNF} \alpha$ for $48 \mathrm{~h}$ to induce CCL11 production. Extracellular media was collected and frozen at $-80^{\circ} \mathrm{C}$ until analysed for CCL11 by ELISA. 
Synergy experiments utilised A549 cells plated and set up as described above. Combinations of procyanidin A2 and procyanidin B2 $(5 \mu \mathrm{M}$ total) were added to cultures for $4 \mathrm{~h}$ prior to the inflammatory insult as described above. Extracellular media was collected and frozen at $-80^{\circ} \mathrm{C}$ until analysed for CCL11 by ELISA.

\subsection{Statistics}

Statistical analysis of data was performed using GraphPad Prism 5 (San Diego, CA, USA) or GenStat: 14th Edition (VSN International, London, UK). Data from CCL11 induction time course and TNF $\alpha$ dose response experiments used GraphPad Prism and were analysed with a 1-way ANOVA with Dunnett's Multiple Comparison test against baseline with statistical significance set at $P<0.05$. All other experiments used GenStat and were analysed with a randomized block design ANOVA using a Fisher's least square difference test with statistical significance set at $P<0.05$. Experiments that did not reach the $P<0.05$ threshold are listed as not significant $(P=\mathrm{NS})$. Statistical significance in figures is denoted as ${ }^{*}=P<0.05,{ }^{* *}=P<0.01,{ }^{* * *}=P<0.0001$.

\section{Results}

\subsection{Optimization of airway epithelial cell bioassay conditions}

The dose response profile of CCL11 production is shown in Fig. 1. Production of CCL11 increased rapidly and reached statistical significance at $2.5 \mathrm{ng} / \mathrm{mL} \mathrm{TNF} \alpha(178 \pm 23 \mathrm{pg} / \mathrm{mL}$ CCL11, $P<0.01)$, then the profile of production slowed from $5 \mathrm{ng} / \mathrm{mL} \mathrm{TNF} \alpha(315 \pm 39 \mathrm{pg} / \mathrm{mL} \mathrm{CCL11})$, and reached a maximum with $40 \mathrm{ng} / \mathrm{mL} \mathrm{TNF} \alpha$ $(515 \pm 6 \mathrm{pg} / \mathrm{mL}$ CCL11) (Fig. 1A). The time course of CCL11 production ranging from $1-72 \mathrm{~h}$ is also shown in Fig. 1. The profile reached a modest plateau between 18-24 h $(298 \pm 24 \mathrm{pg} / \mathrm{mL}$ CCL11 at $18 \mathrm{~h})$, increased to become statistically significant at $48 \mathrm{~h}(505 \pm 166 \mathrm{pg} / \mathrm{mL}$ CCL11, $P<0.05)$, and continued to $706 \pm 316 \mathrm{pg} / \mathrm{mL}$ CCL11 at the last time point of $72 \mathrm{~h}$ (Fig. 1B). From these data we determined that $5 \mathrm{ng} / \mathrm{mL} \mathrm{IL-4} \mathrm{with} 5 \mathrm{ng} / \mathrm{mL}$ $\mathrm{TNF} \alpha$ for $48 \mathrm{~h}$ provided the optimal conditions to induce CCL11 production and additionally allow for the evaluation of effects of procyanidins.
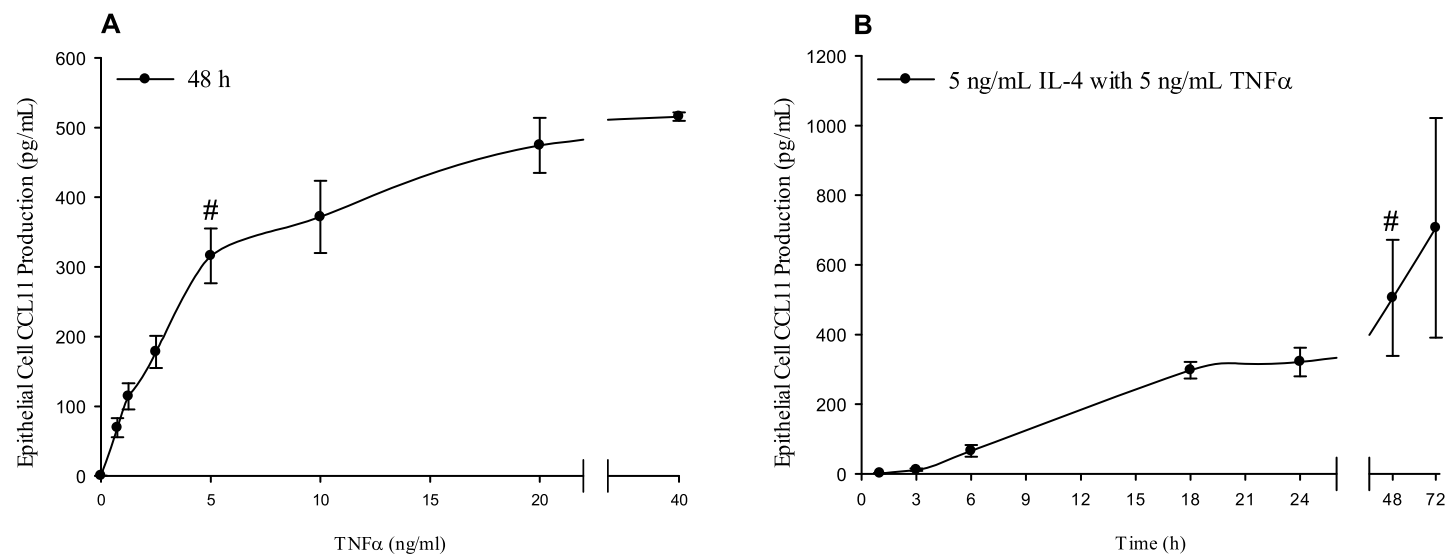

Fig. 1. Airway inflammation cell culture model optimized for inducting CCL11 production in the human lung epithelial cell line A549. A549 cells were incubated with A) $5 \mathrm{ng} / \mathrm{mL}$ IL- 4 with $0.75-40 \mathrm{ng} / \mathrm{mL}$ TNF $\alpha$ for $48 \mathrm{~h}$ or B) $5 \mathrm{ng} / \mathrm{mL}$ IL- 4 with $5 \mathrm{ng} / \mathrm{mL} \mathrm{TNF} \alpha$ for $1-72 \mathrm{~h}$. The culture media supernatants were collected and measured for CCL11 by ELISA. Results are expressed as means \pm SEM, $n=3$ separate experiments. \# indicates the optimal time or dose for inducing CCL11 production. 
A

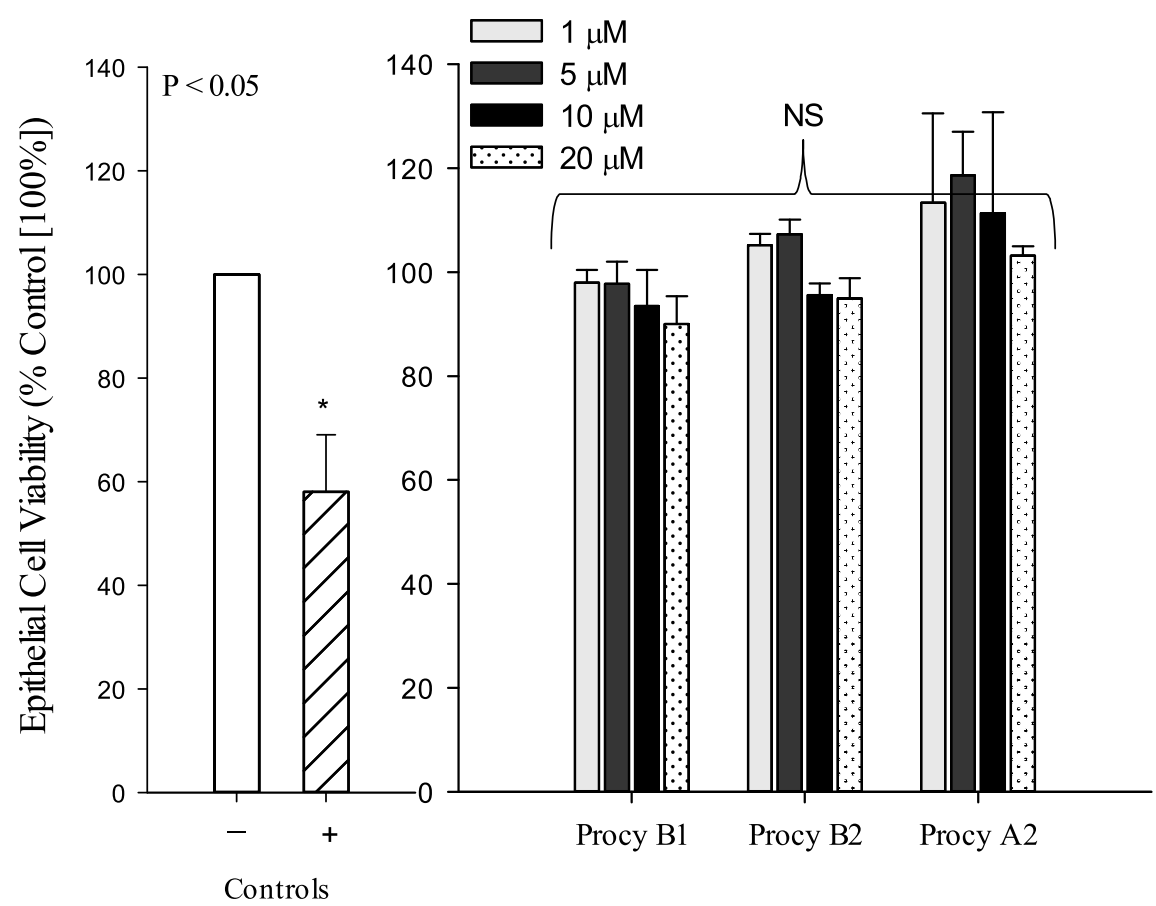

Fig. 2. Effect of procyanidins on viability using a WST-1 Assay. A549 cells were incubated with A) negative (-) control media, or positive (+) control $2.5 \mathrm{mM} \mathrm{H}_{2} \mathrm{O}_{2}$ or B) procyanidin (Procy) B1, B2, or A2 for $6 \mathrm{~h}$ at $1 \mu \mathrm{M}$ (light grey), $5 \mu \mathrm{M}$ (grey), $10 \mu \mathrm{M}$ (black), and $20 \mu \mathrm{M}$ (speckled), washed with PBS and then assayed with WST-1 reagent for cellular viability. Results are expressed as means \pm SEM as a percentage of the negative control [100\%], $n=3$ separate experiments. NS = not statistically significant.

\subsection{Cytotoxicity of procyanidins}

Our primary focus in this study was to investigate modulation of CCL11 by procyanidins. To ensure changes observed after exposure were due to procyanidins and not an artefact of cytotoxicity, we utilized the WST-1 assay to investigate changes in cell metabolism (viability) after exposure to a range of procyanidin concentrations. A positive control of $2.5 \mathrm{mM} \mathrm{H}_{2} \mathrm{O}_{2}$ was established (Fig. 2A) through preliminary experiments (set up as described above for procyanidins) that investigated a range $(1.25-100 \mathrm{mM})$ of $\mathrm{H}_{2} \mathrm{O}_{2}$ concentrations and demonstrated a dose-dependent reduction in viability (data not shown). Exposure to procyanidins B1, B2, and A2 at 1, 5, 10, and $20 \mu \mathrm{M}$ had no statistically significant effect (Fig. 2B) on cell viability compared to control media and were not cytotoxic. Thus, any changes in CCL11 production following procyanidin exposure at these doses is attributed to an effect of the procyanidins and not as a result of toxicity.

\subsection{Evaluation of the effects of procyanidins}

Experiments evaluating the effects of exposure to procyanidins prior to the inflammatory insult revealed distinct differences in efficacy between the three investigated compounds. Experiments included the use of a positive control IFN $\gamma(5.8 \mu \mathrm{M})$, a known inhibitor of eotaxin, that demonstrated a measurable inhibition of CCL11 production (Fig. 3, $P<0.0001$ ). The procyanidin range utilised in our experiments was selected based 


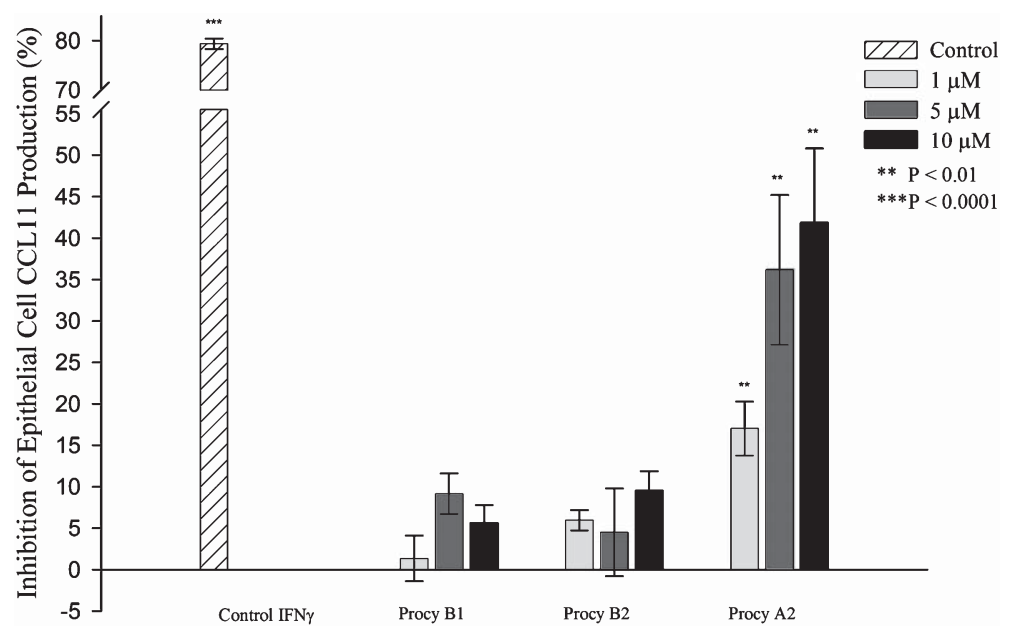

Fig. 3. Inhibition of CCL11 production in A549 cells by procyanidins. A549 cells were exposed to positive control $5.8 \mu \mathrm{M}$ IFN $\gamma$, or procyanidins (Procy) B1, B2, or A2 for $6 \mathrm{~h}$ prior to an inflammatory insult of $5 \mathrm{ng} / \mathrm{mL} \mathrm{IL}-4 \mathrm{with} 5 \mathrm{ng} / \mathrm{mL}$ for $48 \mathrm{~h}$. The culture media supernatants were collected and analysed for CCL11 by ELISA. Results are expressed as mean percent change from DMSO control \pm SEM, $n=3-5$ separate experiments. NS $=$ not statistically significant, ${ }^{* *}=P<0.01$.

on previous work in the literature, the physiological relevance, and demonstrated efficacy on other biomarkers evaluated in other unpublished research by us. [15] We observed that 1,5 and $10 \mu \mathrm{M}$ procyanidin A2 had a statistically significant $(P<0.01)$ inhibitory effect on CCL11 production $(17.0 \pm 3.3,36 \pm 9.0$, and $41.9 \pm 9.0 \%$ inhibition respectively) in a dose-dependent manner when incubated $(6 \mathrm{~h})$ prior to the inflammatory challenge (Fig. 3). Conversely, procyanidin B1 and B2 had no inhibitory effect on CCL11 production at 1, 5, and $10 \mu \mathrm{M}$ $(1.4 \pm 2.8,9.2 \pm 2.5$, and $5.6 \pm 2.2 \%$ inhibition for procyanidin $\mathrm{B} 1$ and $6.0 \pm 1.3,4.5 \pm 5.3,9.5 \pm 2.4 \%$ inhibition for procyanidin B2 respectively) (Fig. 3).

Furthermore, we investigated procyanidins A2 and B2 for possible synergistic relationships. We evaluated the CCL11 inhibitory capacity for a range of concentrations of procyanidins A2 and B2 that together would total $5 \mu \mathrm{M}$ (e.g. $4 \mu \mathrm{M}$ A2 with $1 \mu \mathrm{M} \mathrm{B} 2$ ). We decided to evaluate procyanidin combinations at a total concentration of $5 \mu \mathrm{M}$ because at this dose an inhibitory capacity is likely to be detected (A2 alone inhibition detected, Fig. 3) and this total dose is physiologically relevant. [15] We choose procyanidin B2 as a representative of B-type procyanidins as we had observed it as a moderate inhibitor of another eotaxin isoform (unpublished data). Concentrations of each procyanidin were mixed separately and added together in the cell culture well. For all combinations of procyanidin $\mathrm{A} 2$ and $\mathrm{B} 2$ that totalled $5 \mu \mathrm{M}$, we observed no statistically significant additional inhibition versus procyanidin A2 alone (Table 1).

\section{Discussion}

Evidence supporting the use of plant polyphenolics to improve human health is strong. Scientists are now working toward defining the bioactive molecules that are eliciting effects and through which mechanisms they modulate change [16-18]. There is substantial evidence that procyanidins are capable of modulating key inflammatory pathways in various tissues which has been reviewed elsewhere [7]. Briefly, procyanidins have been demonstrated to modulate signalling pathways that include signal transducers and activators of transcription (STAT), nuclear factor- $\kappa \mathrm{B}(\mathrm{NF}-\kappa \mathrm{B})$, as well as transcription factors in the mitogen-activated protein kinases (MAPK) pathway. 
Table 1

Investigating synergies between procyanidin $\mathrm{A} 2$ and procyanidin $\mathrm{B} 2$

\begin{tabular}{lccccccc}
\hline Procy A2 $(\mu \mathrm{M})$ & 5 & 4 & 3 & 2.5 & 2 & 1 & 0 \\
Procy B2 $(\mu \mathrm{M})$ & 0 & 1 & 2 & 2.5 & 3 & 4 & 5 \\
CCL 11 production & $446.30 \pm$ & $459.53 \pm$ & $465.28 \pm$ & $474.76 \pm$ & $514.04 \pm$ & $514.64 \pm$ & $480.54 \pm$ \\
$(\mathrm{pg} / \mathrm{mL})$ & 17.30 & $24.15^{\mathrm{NS}}$ & $21.56^{\mathrm{NS}}$ & $10.97^{\mathrm{NS}}$ & $16.32^{\mathrm{NS}}$ & $25.31^{\mathrm{NS}}$ & $37.24^{\mathrm{NS}}$ \\
\hline
\end{tabular}

A549 cells were exposed to combinations of procyanidin (procy) A2 and B2 at a range of doses with each combination totalling $5 \mu \mathrm{M}$ for $4 \mathrm{~h}$ prior to an inflammatory insult of $5 \mathrm{ng} / \mathrm{mL}$ IL- 4 with $5 \mathrm{ng} / \mathrm{mL}$ TNF $\alpha$ for $48 \mathrm{~h}$. The culture media supernatants were collected and analysed for CCL11 by ELISA. Results are expressed as epithelial cell CCL11 production (pg/mL) $\pm \mathrm{SEM}, n=3$ separate experiments. NS =not statistically significant vs $5 \mu \mathrm{M}$ A2.

Here, we established an epithelial cell culture model of airway inflammation, and evaluated three different commercially sourced, structurally-defined, pure dimeric procyanidin compounds for their ability to modulate cytokine-induced CCL11 production. Our study provided evidence that procyanidin A2, but not procyanidins of the B-type, inhibits cytokine-induced production of CCL11, a chemokine that facilitates the early-stage infiltration of eosinophils into lung tissue [19]. We further demonstrated that effects were not due to cytotoxicity and our model demonstrated no synergistic interactions between procyanidin A2 and B2 in terms of inhibiting CCL11 production at a combined total of $5 \mu \mathrm{M}$. Our data contribute evidence in support of the continued investigation of A-type procyanidins, and more work in this area with actual A-type procyanidin rich foods (e.g. cranberries) would be a logical next step and could provide further evidence to support possible dietary intervention studies for assisting with the prevention/management of airway inflammation.

Previous work from our group proposed that proanthocyanidins may be the bioactive components responsible for blackcurrant extract-mediated modulation of the production of CCL26 (an isoform of eotaxin) in vitro, using a similar lung epithelial cell culture model of airway inflammation [14]. Furthermore, our group identified that the ratio of specific anthocyanins, another type of polyphenolic compound, in blackcurrant cultivars was an important determinate for influencing the suppression of CCL26 in these cells [20]. Blackcurrants and most other fruits contain procyanidins of the B-type linkage; the present study however, revealed no evidence of modulation of CCL11 by procyanidin B1 or B2. This would suggest that B-type procyanidins are not effective alone but may work in tandem with other bioactives when in a complex polyphenolic mixture such as fruit extracts to elicit a change in airway inflammation. However, our knowledge is limited on the exact role, if any, B-type procyanidins play in assisting the inhibition of airway inflammation.

Our evidence is promising, but should not be extrapolated at this time as further investigation is needed to determine if A-type procyanidins would have an effect on airway inflammation in vivo. The model system utilized in this work was designed to emulate distribution of procyanidins to the airway epithelium through systemic circulation; another interesting approach would be to investigate A-type procyanidins applied as aerosols, mimicking inhalation. This could be modelled experimentally by procyanidin exposure directly to the dorsal surface of an air-liquid interface epithelial cell culture. The cell culture model used here in this study attempted to mimic Th2 cytokine mediated responses, which are relevant to the allergic asthma endo-type [3]. Asthma is now understood to be a syndrome that can be caused by multiple biological mechanisms, which present in the clinic as differences in immunological features, histology, morphological characteristic of the tissue, as well as differences in responses to treatment [3]. The prominent Th2 cytokines in allergic asthma responses are IL-4 and IL-13, both of which play roles in asthma pathogenesis [4]. IL-4 signalling can utilize two interleukin receptors, the type I IL-4R which can only bind IL-4 and the type II IL-4R, which can also bind IL-13. In the present work we used IL-4 to induce epithelial cell CCL11 production as it has been suggested that the type I IL-4R is necessary for eosinophilia [21]. It could be useful in future work to investigate the modulation of other biomarkers due to an inflammatory insult by IL-13, as IL-13 binding to its own unique receptor chain, IL-13R $\alpha 2$, 
may have a distinct role in airway inflammation [22]. Our model did not include the potentially large impact of the gut microbial community. There is currently considerable controversy on the bioavailability of dimeric procyanidins. Some evidence suggests that dimeric proanthocyanidin compounds found in foods, referred to as the parent compounds, would be able to enter systemic circulation and reach peripheral tissues in the original form [23], whereas other research suggests it is unlikely procyanidins of any size could reach tissues, and that any polyphenolic compounds available at tissues would be metabolites of the gut microbial community and/or subject to phase two conjugation [10,24]. Epithelial cells of both the gastrointestinal tract and the lung are capable of drug metabolism and in our in vitro model it is unknown if the bioactivity measured is due to an epithelial cell metabolite or the parent compound, procyanidin A2. Our studies warrant further investigation of the effects of procyanidins in an in vitro model system of inflammation to decipher mechanism of action. Furthermore, investigation is needed in a whole body system of either animal or human to determine if the use of A-type procyanidins could support the management of inappropriate airway inflammation found in allergic asthma.

In our study, only procyanidin A2 was able to inhibit CCL11 production in the cell culture system, procyanidins B1 and B2 were not able to modulate CCL11 production significantly. Other work by our group has suggested that B-type procyanidins, in a complex mixture from fruit extracts, may inhibit other isoforms of eotaxin and thus we investigated the possibility of a synergistic relationship between A-type and B-type procyanidins for CCL11 inhibition. However, there were no significant differences observed in our experimental model when inhibition of CCL11 production by a combination of A- and B-type procyanidins totalling $5 \mu \mathrm{M}$ was compared to inhibition of CCL11 production by $5 \mu \mathrm{M}$ procyanidin A2 alone. Polyphenolic compounds evolved in plants to assist in coping with stresses such as drought, excess UV exposure and/or pathogenic invasion, and yet there isn't a fruit identified with high concentrations of both A-type and B-type procyanidins [10]. Thus it could be speculated that these two different types of procyanidins may result in the same terminal protection of the plant from stress but through different pathways. It has been shown that B-type procyanidins are capable of forming structurally unique hydrogen bonds with subunits of NF- $\mathrm{B}$ and are thus capable of inhibiting transcription of genes controlled by the NF-кB transcription factor [25]. Furthermore, Matsukura et al. demonstrated that the mechanism for CCL11 expression, when induced by TNF $\alpha$ and IL-4, is via the activation of the transcription factors, NF-кB and STAT6 [26]. As A-type procyanidins do not bind to NF-кB in the same manner it can be speculated that A-type procyanidin inhibition of CCL11 would most likely be mediated through STAT6.

The greatest potential for a dietary intervention to improve asthma outcomes in people would be to modulate multiple aspects of the condition, as asthma aetiology is not only characterised by inappropriate airway inflammation and there is data to suggest a dissociation between airway hyper-responsiveness and the other pathophysiological features of asthma [27]. The in vitro data presented here supports the hypothesis that procyanidins maybe be able to assist with the prevention of airway inflammation. More research is warranted to investigate the effect of procyanidins on biomarkers that are involved in the other aspects of asthma progression such as hyperresponsive bronchiole constrictions, or lung tissue remodelling. Understanding the potential capabilities of each procyanidin would allow for the focused use of single or multiple procyanidins in fresh and processed foods as a natural means to prevent inflammatory illness, limit the use of pharmaceutical intervention, and improve human health.

\section{Acknowledgments}

The authors would like to thank Dr Andrew McLachlan for statistical assistance. Educational resources were provided to S.L.C. by the School of Food and Nutrition, College of Health, Massey University. This work was funded by the New Zealand Ministry of Business, Innovation, and Employment contract (C11X1002) awarded to The New Zealand Institute for Plant \& Food Research Ltd. 


\section{Conflict of interest}

The authors have declared no conflict of interest.

\section{References}

[1] Singh A, Holvoet S, Mercenier A. Dietary polyphenols in the prevention and treatment of allergic diseases. Clin Exp Allergy. 2011;41(10):1346-59. DOI: 10.1111/j.1365-2222.2011.03773.x

[2] Nyanhanda T, Gould EM, Hurst RD. Plant-derived foods for the attenuation of allergic airway inflammation. Curr Pharm Des. 2014;20(6):869-78. DOI: 10.2174/13816128113199990056

[3] Ishmael FT. The inflammatory response in the pathogenesis of asthma. J Am Osteopath Assoc. 2011;111(11 Suppl 7):S11-7.

[4] Lambrecht BN, Hammad H. The airway epithelium in asthma. Nat Med. 2012;18(5):684-92. DOI: 10.1038/nm.2737

[5] Rokudai A, Terui Y, Kuniyoshi R, Mishima Y, Mishima Y, Aizu-Yokota E, et al. Differential regulation of eotaxin-1/CCL11 and eotaxin-3/CCL26 production by the TNF-alpha and IL-4 stimulated human lung fibroblast. Biol Pharm Bull. 2006;29(6):1102-9. DOI: $10.1248 /$ bpb.29.1102

[6] Xie DY, Dixon RA. Proanthocyanidin biosynthesis - still more questions than answers? Phytochemistry. 2005;66(18):2127-44. DOI: 10.1016/j.phytochem.2005.01.008

[7] Coleman SL, Hurst RD, Sawyer GM, Kruger MC. Fruit Procyanidins: Modulating Inflammation to Promote Health. In: Sullivan I, editor. Proanthocyanidins: Food Sources, Antioxidant Properties, and Health Benefits. New York: Nova Science; 2015, pp. 73-97.

[8] Rzeppa S, Von Bargen C, Bittner K, Humpf H-U. Analysis of flavan-3-ols and procyanidins in food samples by reversed phase high-performance liquid chromatography coupled to electrospray ionization tandem mass spectrometry (RP-HPLC-ESI-MS/MS). J Agric Food Chem. 2011;59(19):10594-603. DOI: 10.1021/jf202697j

[9] Treutter D. Managing phenol contents in crop plants by phytochemical farming and breeding-visions and constraints. Int J Mol Sci. 2010;11(3):807-57. DOI: 10.3390/ijms11030807

[10] Joven J, Micol V, Segura-Carretero A, Alonso-Villaverde C, Menendez JA, Bioactive Food Components Platform. Polyphenols and the modulation of gene expression pathways: Can we eat our way out of the danger of chronic disease? Crit Rev Food Sci Nutr. 2014;54(8):985-1001. DOI: 10.1080/10408398.2011.621772

[11] Cocco C, Magnani S, Maltoni ML, Quacquarelli I, Cacchi M, Antunes LEC, et al. Effects of site and genotype on strawberry fruits quality traits and bioactive compounds. J Berry Res. 2015;5(3):145-55. DOI: 10.3233/jbr-150098

[12] Usual Dietary Intakes: Food Intakes, U.S. Population, 2007-10. [accessed Accessed February 2, 2016. Available from: http://epi.grants.cancer.gov/diet/usualintakes/pop/2007-10/.

[13] Tan AS, Berridge MV. Superoxide produced by activated neutrophils efficiently reduces the tetrazolium salt, WST-1 to produce a soluble formazan: A simple colorimetric assay for measuring respiratory burst activation and for screening anti-inflammatory agents. J Immunol Methods. 2000;238(1-2):59-68. DOI: 10.1016/S0022-1759(00)00156-3

[14] Hurst SM, McGhie TK, Cooney JM, Jensen DJ, Gould EM, Lyall KA, et al. Blackcurrant proanthocyanidins augment IFN-gammainduced suppression of IL-4 stimulated CCL26 secretion in alveolar epithelial cells. Mol Nutr Food Res. 2010;54:S159-70. DOI: 10.1002/mnfr.200900297

[15] Serra A, Macia A, Romero M-P, Salvado M-J, Bustos M, Fernandez-Larrea J, et al. Determination of procyanidins and their metabolites in plasma samples by improved liquid chromatography-tandem mass spectrometry. J Chromatogr B. 2009;877(11-12):1169-76. DOI: 10.1016/j.jchromb.2009.03.005

[16] Giampieri F, Alvarez-Suarez JM, Battino M. Strawberry and human health: Effects beyond antioxidant activity. J Agric Food Chem. 2014;62(18):3867-76. DOI: 10.1021/jf405455n

[17] Giampieri F, Tulipani S, Alvarez-Suarez JM, Quiles JL, Mezzetti B, Battino M. The strawberry: Composition, nutritional quality, and impact on human health. Nutrition. 2012;28(1):9-19. DOI: 10.1016/j.nut.2011.08.009

[18] Denis MC, Furtos A, Dudonne S, Montoudis A, Garofalo C, Desjardins Y, et al. Apple peel polyphenols and their beneficial actions on oxidative stress and inflammation. PLoS ONE. 2013;8(1). DOI: 10.1371/journal.pone.0053725

[19] Ravensberg AJ, Ricciardolo FLM, van Schadewijk A, Rabe KF, Sterk PJ, Hiemstra PS, et al. Eotaxin-2 and eotaxin-3 expression is associated with persistent eosinophilic bronchial inflammation in patients with asthma after allergen challenge. J Allergy Clin Immunol. 2005;115(4):779-85. DOI: 10.1016/j.jaci.2004.11.045

[20] Nyanhanda T, Gould EM, McGhie T, Shaw OM, Harper JL, Hurst RD. Blackcurrant cultivar polyphenolic extracts suppress CCL26 secretion from alveolar epithelial cells. Food Funct. 2014;5(4):671-7. DOI: 10.1039/c3fo60568j

[21] Munitz A, Brandt EB, Mingler M, Finkelmant FD, Rothenberg ME. Distinct roles for IL-13 and IL-4 via IL-13 receptor alpha 1 and the type II IL-4 receptor in asthma pathogenesis. Proc Natl Acad Sci U S A. 2008;105(20):7240-5. DOI: 10.1073/pnas.0802465105 
[22] Gour N, Wills-Karp M. IL-4 and IL-13 signaling in allergic airway disease. Cytokine. 2015;75(1):68-78. DOI: 10.1016/j. cyto.2015.05.014

[23] Ou K, Gu L. Absorption and metabolism of proanthocyanidins. J Funct Food. 2014;7:43-53. DOI: 10.1016/j.jff.2013.08.004

[24] Del Rio D, Borges G, Crozier A. Berry flavonoids and phenolics: Bioavailability and evidence of protective effects. Br J Nutr. 2010;104:S67-90. DOI: 10.1017/s0007114510003958

[25] Mackenzie GG, Delfino JM, Keen CL, Fraga CG, Oteiza PI. Dimeric procyanidins are inhibitors of NF-kappa B-DNA binding. Biochem Pharmacol. 2009;78(9):1252-62. DOI: 10.1016/j.bcp.2009.06.111

[26] Matsukura S, Stellato C, Plitt JR, Bickel C, Miura K, Georas SN, et al. Activation of eotaxin gene transcription by NF-kappa B and STAT6 in human airway epithelial cells. J Immunol. 1999;163(12):6876-83.

[27] Chapman DG, Tully JE, Nolin JD, Janssen-Heininger YM, Irvin CG. Animal models of allergic airways disease: Where are we and where to next? J Cell Biochem. 2014;115(12):2055-64. DOI: 10.1002/jcb.24881 